\title{
Exploring the Dynamics of Bound Water in Nylon Polymers with Terahertz Spectroscopy
}

Hiromichi Hoshina ${ }^{1}$, Takuro Kanemura ${ }^{1}$, Chiko Otani ${ }^{1}$ and Michael Ruggiero ${ }^{2}$

${ }^{1}$ RIKEN, Center for Advanced Photonics, 519-1399 Aramaki-Aoba, Aoba-ku, Sendai, Miyagi, 9800845 Japan

${ }^{2}$ Department of Chemistry, University of Vermont, 82 University Place, Burlington, Vermont 05405 USA 


\section{Abstract:}

Terahertz (THz) spectroscopy was used to observe adsorbed water structure and dynamics within polymer films, ultimately providing a strong rationale for the observed rates of water desorption. The THz absorption spectra of nylon-6 films undergoes drastic changes during the hydration and drying process. Additionally, the structural change from $\gamma$ to $\alpha$ crystals, induced by the hydration, was observed by the characteristic band of $\alpha$-nylon- 6 at $6.5 \mathrm{THz}$. Importantly, the THz spectra of adsorbed water, as well as deuterated water, within in the nylon films were observed by the continuous measurement of $\alpha$-nylon during dehydration. The differential spectra clearly show three absorption bands of water molecules named Peak I, II and III, which behaved differently between the $\mathrm{H}_{2} \mathrm{O}$ and $\mathrm{D}_{2} \mathrm{O}$ materials. The spectra were assigned using a combination of ab initio molecular dynamics simulations and solid-state density functional theory calculations, and were compared to previous spectral assignments of bulk water. The results show that the inclusion of $\mathrm{H}_{2} \mathrm{O}$ and $\mathrm{D}_{2} \mathrm{O}$ into polymer films results in a distinct set of spectral features that, while similar in frequencies to the dynamics of bulk water, represent significantly different motions owing to the unique chemical environment within the material. These results highlight the significant utility of using $\mathrm{THz}$ spectroscopy to study the hydration dynamics and spectral signature of bound water in this important class of materials. 
Keywords

Terahertz Spectroscopy, polymer, nylon 6, polyamide 


\section{- INTRODUCTION}

Due to the rapid developments of technologies related to terahertz $(\mathrm{THz})$ spectroscopy, quick and precise measurements have become possible in the THz frequency region $(0.1-10 \mathrm{THz}$ or 3

- $333 \mathrm{~cm}^{-1}$ ), in which organic materials show characteristic spectra (so-called fingerprint spectra) ${ }^{1,2}$ Since those absorption bands are mainly due to the intermolecular vibrational motion, ${ }^{3,4} \mathrm{THz}$ spectroscopy provides detailed information about intermolecular structures and intermolecular interactions such as hydrogen bondings. ${ }^{5-8}$ Especially for macromolecules, $\mathrm{THz}$ spectra give direct information about higher order conformation and hydrogen bonding networks, all of which play an important role for their physical and chemical properties. Therefore, $\mathrm{THz}$ spectroscopy is poised to become a powerful tool for research in polymer science.

Low-frequency vibrational spectroscopy of polymers have been studied in far-infrared and $\mathrm{THz}$ frequency regions, and the relationship between $\mathrm{THz}$ spectral features and polymer structures and properties has been studied. ${ }^{9-15}$ In our previous studies, the THz spectra of polyesters ${ }^{16-20}$ and polyamides ${ }^{21,22}$ were studied. In order to assign the spectra, the vibrational dipole moments were determined using polarized $\mathrm{THz}$ spectroscopy on stretched samples, ${ }^{16,17}$ and the experimental THz spectra was compared with low-frequency Raman spectra in order to uncover the origins of the vibrational modes. ${ }^{17,18}$ Additionally, quantum mechanical calculations were applied to obtain low-frequency vibrational modes. ${ }^{18-20}$ In this regard, the coupling of $\mathrm{THz}$ 
spectral measurements with $a b$ initio simulations provides unparalleled insight into the forces and dynamics that drive the properties of materials. Advances in computational resources have permitted increasingly large calculations to be performed, which has recently proven to be valuable for a wide-range of applications, including phase-transformation phenomena, organic semiconducting efficiency, and pharmaceutical stability, to name a few. ${ }^{23-25}$ In those studies, the structural information obtained by $\mathrm{THz}$ spectroscopy is in good agreement with those obtained by conventional methods, such as differential scanning calorimetry (DSC), NMR, and X-ray diffraction measurements. Moreover, $\mathrm{THz}$ spectroscopy shows great advantages for in situ spectroscopy, such as time-lapse monitoring of the isothermal crystallization ${ }^{17,26}$ or the imaging of the spatial distribution of higher order structures in the samples. ${ }^{27}$

Another advantage of $\mathrm{THz}$ spectroscopy is the high sensitivity to water molecules. Water molecules show intense absorption in $\mathrm{THz}$ frequencies due to their large permanent dipole moment. ${ }^{28-31}$ Therefore, even small amounts of water contamination in the samples can be detected by THz spectroscopy. For macromolecules, the THz spectra of adsorbed water has long been studied, along with the spectral change with the hydration has been studied. ${ }^{32-37}$ In most of those studies, measurements have been performed using THz-TDS or dielectric spectroscopy, and only acquired below $3 \mathrm{THz}$, where only broad spectral changes were observed. ${ }^{30,31}$ On the other hand, $\mathrm{THz}$ spectra above $4 \mathrm{THz}$ provides significantly more detailed information about water 
dynamics, because in those frequencies, intermolecular vibrational motion ( $\mathrm{S}$ band at $5.2 \mathrm{THz}$ ) and libration motion $\left(\mathrm{L}_{1}\right.$ and $\mathrm{L}_{2}$ bands at 12 and $\left.20 \mathrm{THz}\right)$ are observed. ${ }^{28}$ In our previous paper, $\mathrm{THz}$ spectra of hydrated polymer films were measured from 1-9 THz, and dynamics of bound water were discussed by the intensity ratio between vibrational and librational modes. ${ }^{38}$

In this study, the hydration of nylon-6 films was observed with THz spectroscopy. Nylon-6 is a crystalline polymer used in various industrial applications. The structure of nylon- 6 consists of amide groups that form a $\mathrm{NH} \cdots \mathrm{CO}$ hydrogen bonding network between polymer chains, which makes nylon-6 hydrophilic. In 1960's, Puffr et al. proposed the structure model of water in polyamides as three types of water molecules. ${ }^{39}$ The first type forms double hydrogen bonds with the $\mathrm{CO}$ groups, the second forms hydrogen bonds with both the $\mathrm{CO}$ and $\mathrm{NH}$ groups, and the third exhibits no hydrogen bonds with nylon. ${ }^{39}$ After that, the dynamics and structure of adsorbed water in nylon-6 has been studied using neutron and X-ray scattering, ${ }^{40-44} \mathrm{NMR},{ }^{45,46}$ infrared spectroscopy ${ }^{40,47,48}$ and ab initio molecular dynamics (AIMD) simulations, ${ }^{49}$ but never with lowfrequency vibrational spectroscopy, which is much more sensitive to intermolecular interactions than other techniques.

In this paper, the THz spectra of hydration of nylon- 6 is first observed. The nylon- 6 samples were hydrated by both $\mathrm{H}_{2} \mathrm{O}$ and $\mathrm{D}_{2} \mathrm{O}$, and the spectra corresponding to bound water was observed by taking the difference between the hydrated and dehydrated samples. The spectral features of 
the hydrated water were resolved into three Gaussian functions. For the assignment of $\mathrm{THz}$ spectra, ab initio molecular dynamics (AIMD) and solid-state density functional theory (DFT) simulations were performed. Through the intensity change of the observed bands, the dynamics of adsorbed water molecules is discussed. Finally, the insight gained through the assignment of the low-frequency spectra provides a concise description for the rate of hydration and dehydration in these materials, highlighting the utility of $\mathrm{THz}$ spectroscopy for characterizing such phenomena.

\section{- RESULTS AND DISCUSSION}

\section{Crystallization of nylon-6 induced by hydration.}

In amorphous polymers that are capable of hydrogen bonding, adsorption of water molecules acts as a plasticizer to lower the glass transition temperature $\left(\mathrm{T}_{\mathrm{g}}\right)$. When stored below $\mathrm{T}_{\mathrm{g}}$, the movement of the individual polymer chains is suppressed, which results in metastable to stable structures. However, when the $T_{g}$ of hydrated nylon- 6 is lower than the environmental temperature, increased molecular mobility results in an decrease in the amorphous structure and a concomitant increase in the $\alpha$-crystalline structure. ${ }^{40}$ Thus, it is of critical importance that the role of water on the structure and dynamics of amorphous polymers be uncovered in order to more effectively use such materials in advanced applications.

Figure 1 shows the terahertz absorption spectra of nylon- 6 stored in different relative humidities. 
The $\mathrm{THz}$ spectra are measured after (i) the samples were vacuum dried at $40{ }^{\circ} \mathrm{C}$ for 1 week, (ii) the samples were stored in the hydration chamber for 1 week, and (iii) after the samples were vacuum dried again at $40{ }^{\circ} \mathrm{C}$ for 1 week. The humidity of the hydration chamber was controlled by a saturated solution of (a) $\mathrm{KCOOCH}_{3}(22 \% \mathrm{RH})$ and (b) $\mathrm{NaCl}(76 \% \mathrm{RH})$, respectively. The initial spectra (i) show no absorption bands at $6.5 \mathrm{THz}$, and shows a similar spectral shape as $\gamma$ nylon-6, as observed in our previous stidues. ${ }^{21,22}$ The THz spectra clearly show that our sample preparation results in nylon-6 films containing both amorphous and $\gamma$-crystalline structures.

After the samples are hydrated for 1 week (ii), the absorbance increases in 3-9 THz due to the water molecules adsorbed in the samples. In addition, a band structure appears at $6.5 \mathrm{THz}$ in the spectra of (b). This band is known as a characteristic band for $\alpha$-nylon- $6,{ }^{21,22}$ and this band does not disappear after the samples are vacuum dried again. This result shows that the $\gamma \rightarrow \alpha$ crystalline change is induced by the hydration in the $76 \% \mathrm{RH}$ chamber. In this study, cycles of drying and hydration process are performed for each sample in the vacuum dried chamber $\left(40^{\circ} \mathrm{C}\right)$ and the hydration chamber $(100 \% \mathrm{RH})$, to make their macromolecular structure in equilibrium for the water adsorption. 
(a)

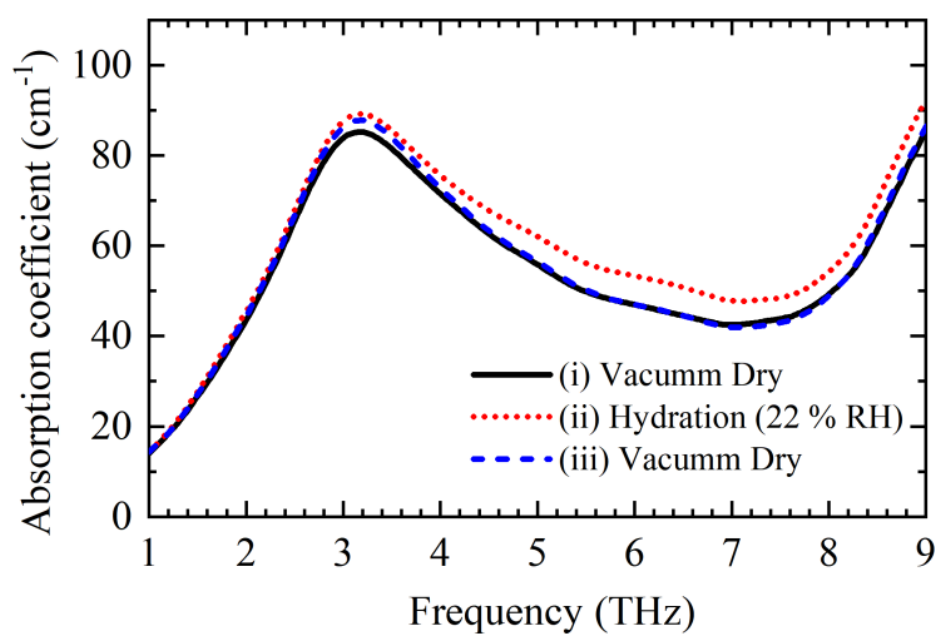

(b)

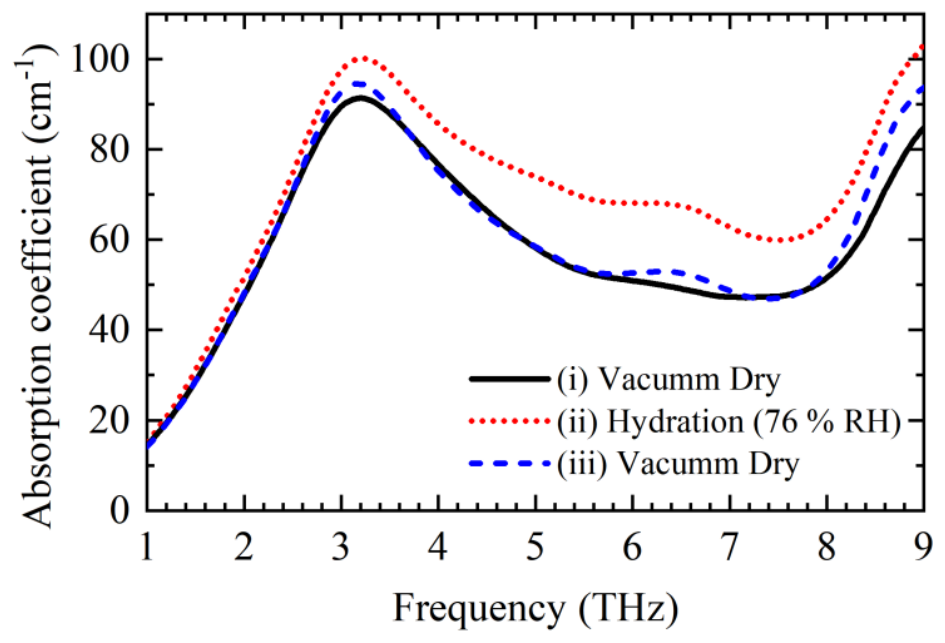

Figure 1 Terahertz absorption spectra of nylon-6 stored in different humidity. (a) Vacuum dried nylon-6 film was stored in $22 \% \mathrm{RH}$ for 1 week and then vacuum dried for 1 week. (b) Vacuum dried nylon- 6 film was stored in $76 \%$ RH for 1 week and then vacuum dried for 1 week.

THz spectra of bound water in nylon 6 films during desorption. THz spectra of bound water in the nylon 6 films during drying the sample were measured. The nylon samples are 
hydrated in the chamber saturated with water $\left(\mathrm{H}_{2} \mathrm{O}\right.$ or $\left.\mathrm{D}_{2} \mathrm{O}\right)$ vapor $(100 \% \mathrm{RH})$ for 1 week and then $\mathrm{THz}$ spectra were measured continuously in the sample chamber of the Fourier transform far-infrared (FT-FIR) spectrometer, which was purged by continuous flow of nitrogen gas (20 $\mathrm{L} / \mathrm{min}$ ). The hydrated samples were dried out continuously, and thus THz spectra of nylon- 6 with different water content are obtained. First, weight loss of the sample in the chamber was measured to evaluate water content in the sample. Figure 2 shows the temporal change of the water content in the nylon- 6 sample as a function of time. The percentage of the water content was least squares fitted by a double exponential function as,

$y=5.1 \exp (-t / 13.3)+5.8 \exp (-t / 193)$

where $t$ is time in minutes and $y$ is the water content in weight $\%$, respectively. The initial water content of the sample is approximately $10 \%$. The fast component of the water desorption occurs in 10 minutes, and slow desorption occurs in 3 hours. 


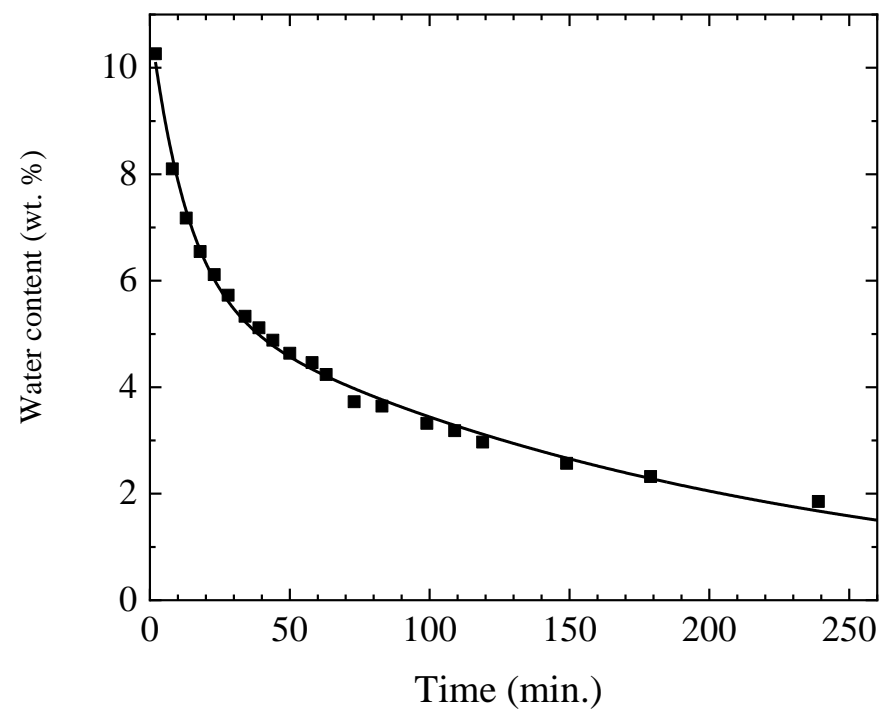

Figure 2 Water content of the nylon- 6 in the sample chamber of FT-IR. The sample was hydrated in the $100 \%$ humidity for 2 weeks and dried in the sample chamber.

Figure 3 (a) shows the $\mathrm{THz}$ absorption spectra of nylon- 6 before hydration (black line) and those after hydration by $\mathrm{H}_{2} \mathrm{O}$. The samples are dried in the chamber and $\mathrm{THz}$ spectra were measured every 10 minutes. Figure 3 (b) shows the differential spectra $(\Delta \alpha)$ obtained by subtracting the spectra before and after hydration. ${ }^{38}$ The spectral features that appear in $\Delta \alpha$ are completely different from those of nylon- 6 , as $\Delta \alpha$ is mainly due to the absorbed water molecules in the nylon films. The same experiment was performed for $\mathrm{D}_{2} \mathrm{O}$ and the results were compared. Figure 4 shows THz absorption spectra and $\Delta \alpha$ of the nylon- 6 film hydrated by $\mathrm{D}_{2} \mathrm{O}$. The $\Delta \alpha$ pattern shows a different shape from those of $\mathrm{H}_{2} \mathrm{O}$. In the spectra of $\mathrm{D}_{2} \mathrm{O}$ samples, $\Delta \alpha$ shows 
higher absorbance in the higher frequencies.

(a)

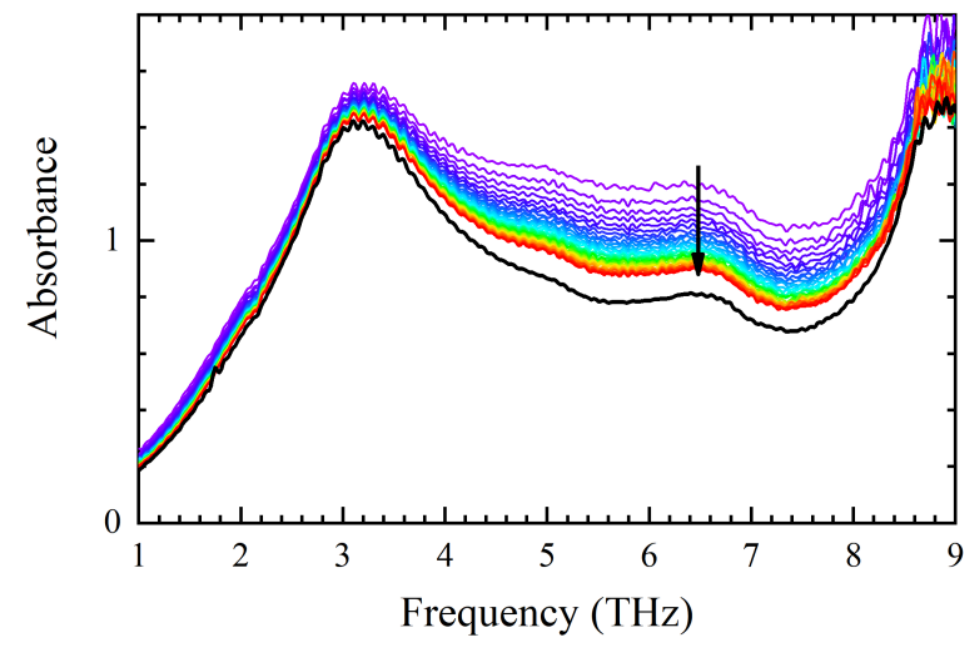

(b)

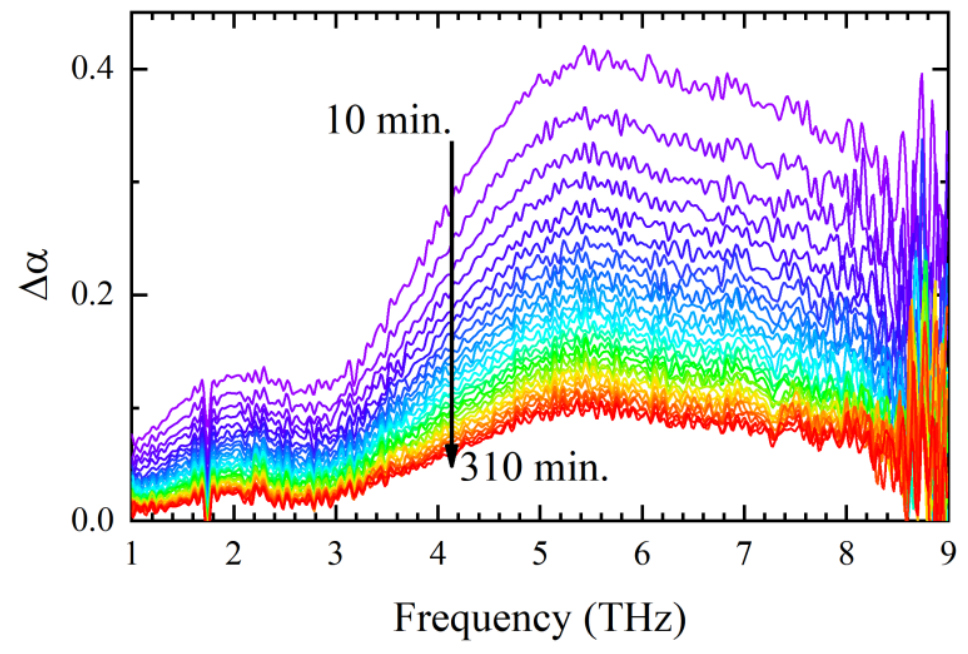

Figure 3 (a) THz absorption spectra of nylon-6 before hydration (Black line) and after hydrated by $\mathrm{H}_{2} \mathrm{O}$. Spectra was dried in the chamber for 10 to 310 minutes as shown in the solid arrow. (b) Differential spectra " $\Delta \alpha$ " obtained by subtracting spectra before and after hydration. 

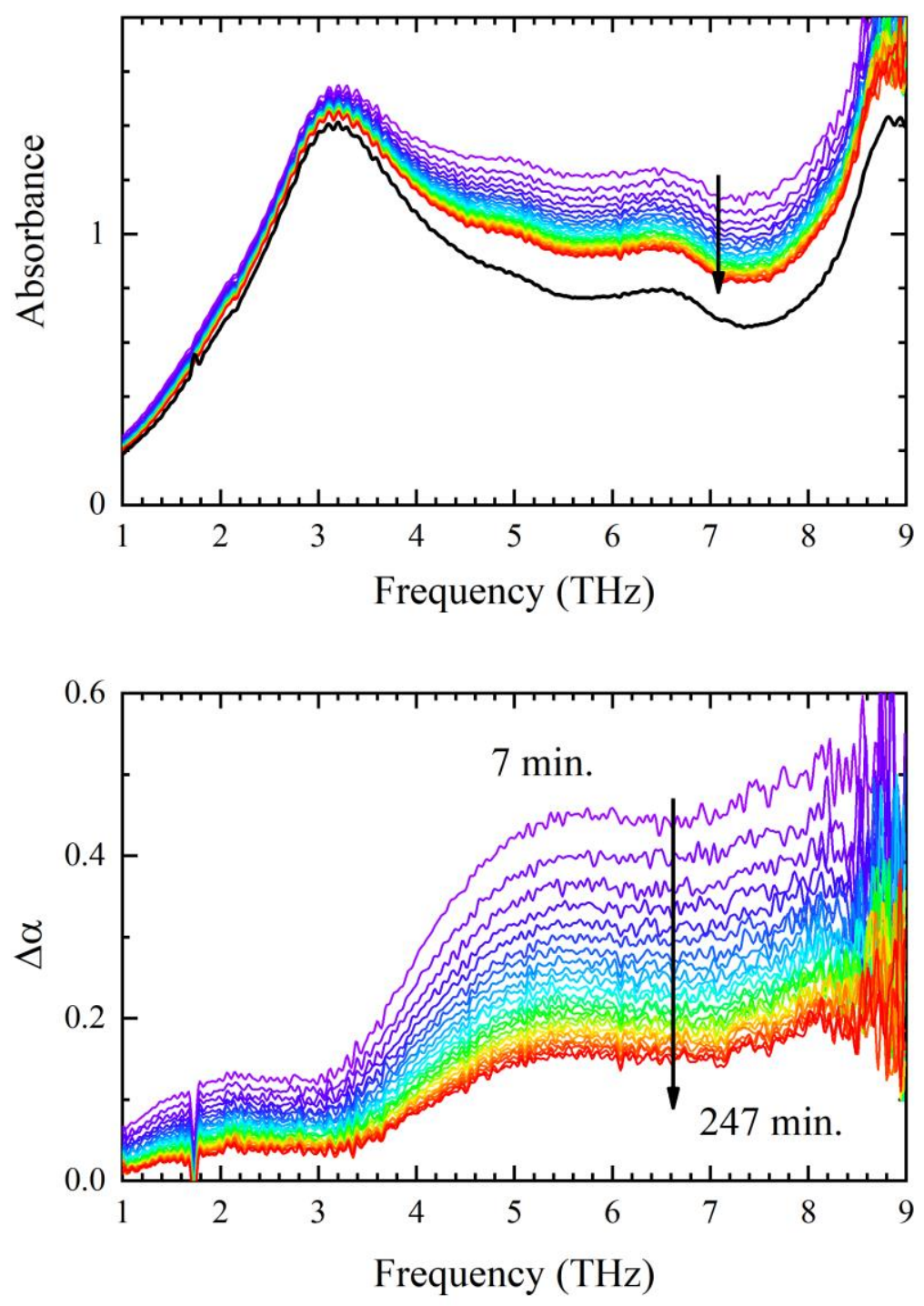

Figure 4 (a) THz absorption spectra of nylon-6 before hydration (Black line) and after hydrated by $\mathrm{D}_{2} \mathrm{O}$. Spectra was dried in the chamber for 7 to 247 minutes as shown in the solid arrow. (b) Differential spectra " $\Delta \alpha$ " obtained by subtracting spectra before and after hydration. 
Absorption bands of bound water in the nylon 6 films. To separate the overlapped bands, spectra of $\Delta \alpha$ were fit with Gaussian functions. The black lines in Figure 5 (a) show $\Delta \alpha$ of $\mathrm{H}_{2} \mathrm{O}$ at 10 minutes, and (b) $\mathrm{D}_{2} \mathrm{O}$ at 7 minutes, respectively. Each spectrum is fit by three Gaussian functions, labeled as Peak I, II and III, as shown in the colored lines. The residual of the fitting is shown in the upper panel of each graph. Table I shows the center frequency, full width half maximum (FWHM) and peak height of the fitted Gaussian functions, respectively.

By comparing the obtained parameters between $\mathrm{H}_{2} \mathrm{O}$ and $\mathrm{D}_{2} \mathrm{O}$, the origin of the vibrational modes can be deduced. First, the center frequencies of Peak II is close to the frequency of the S band of the liquid water, which is known as the intermolecular stretching vibration between water molecules with tetrahedral conformation. ${ }^{28,31}$ Although the exact value of the oscillating frequencies differs in the references, the center frequency of this mode always red-shifts by $1-3 \%$ when changing from $\mathrm{H}_{2} \mathrm{O}$ to $\mathrm{D}_{2} \mathrm{O}{ }^{28,31}$ The obtained center frequencies of the Peak II also shows a similar isotope shift of about $1 \%$. Therefore, we initially assign this mode as $\mathrm{S}$ band of adsorbed water.

The center frequencies of Peak I are close in frequency to the intermolecular bending vibration mode of liquid water. Since this vibration is Raman active, the bands are mainly observed by Raman and hyper Raman spectroscopy. ${ }^{50-52}$ The center band position is $1.62 \mathrm{THz}\left(\mathrm{H}_{2} \mathrm{O}\right)$ and 1.56 $\mathrm{THz}\left(\mathrm{D}_{2} \mathrm{O}\right)$, respectively. ${ }^{51}$ In the THz absorption spectra of the liquid water, the band intensity is 
weak and overlapped with the other absorption features. Recently, Shiraga et al. succeeded in obtaining the band frequencies by refined fitting model with the stochastic frequency modulation mode and obtained the center frequencies as $1.34 \mathrm{THz}\left(\mathrm{H}_{2} \mathrm{O}\right)$ and $1.33 \mathrm{THz}\left(\mathrm{D}_{2} \mathrm{O}\right)$, respectively. Both in the Raman and THz spectroscopy, the center frequency of $\mathrm{H}_{2} \mathrm{O}$ is higher than that of $\mathrm{D}_{2} \mathrm{O}$. However, the isotope shift is opposite for Peak I. The center frequency of $\mathrm{D}_{2} \mathrm{O}$ is $4 \%$ higher than that of $\mathrm{H}_{2} \mathrm{O}$. Therefore, Peak I is not simple intermolecular bending motion between water molecules, but strongly coupled vibration of water bending mode and the vibration of the nylon chains.

Peak III, observed around $8 \mathrm{THz}$, shows large differences in the fitting parameters between $\mathrm{H}_{2} \mathrm{O}$ and $\mathrm{D}_{2} \mathrm{O}$. In this frequency region, Peak III and the $\mathrm{L}_{1}$ band are overlapped and cannot be resolved by the least squares fitting. It is reported that the center frequency of the $\mathrm{L}_{1}$ band is 11.9 THz for $\mathrm{H}_{2} \mathrm{O}$ and $9.5 \mathrm{THz}$ for $\mathrm{D}_{2} \mathrm{O}$, respectively. ${ }^{28}$ The tale of the $\mathrm{L}_{1}$ band must be overlapping with Peak III and resulted in changing their lineshapes. As a result, the fitting parameters of $\mathrm{D}_{2} \mathrm{O}$ become higher intensity and higher frequency than those of $\mathrm{H}_{2} \mathrm{O}$. The original mode of the Peak III, located around $8 \mathrm{THz}$, has no corresponding vibrational mode in the spectra of liquid water. Therefore, the origin of Peak III is not vibration between water molecules but the collective vibration of water molecules and polymer chains interacting with strong hydrogen bondings. 
(a)

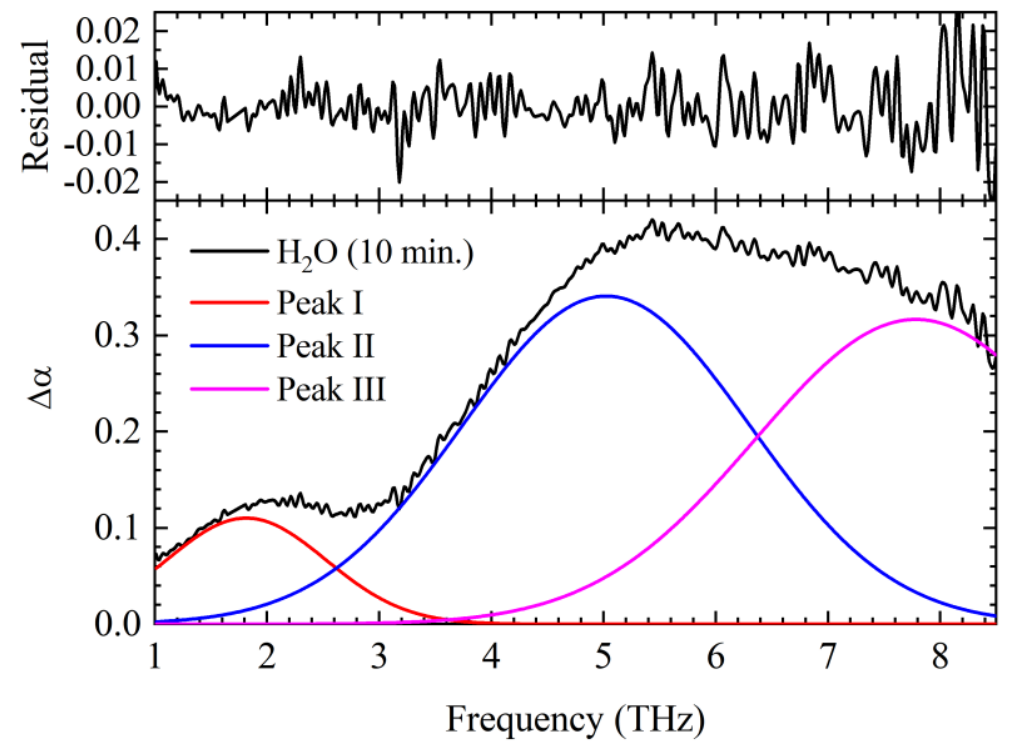

(b)

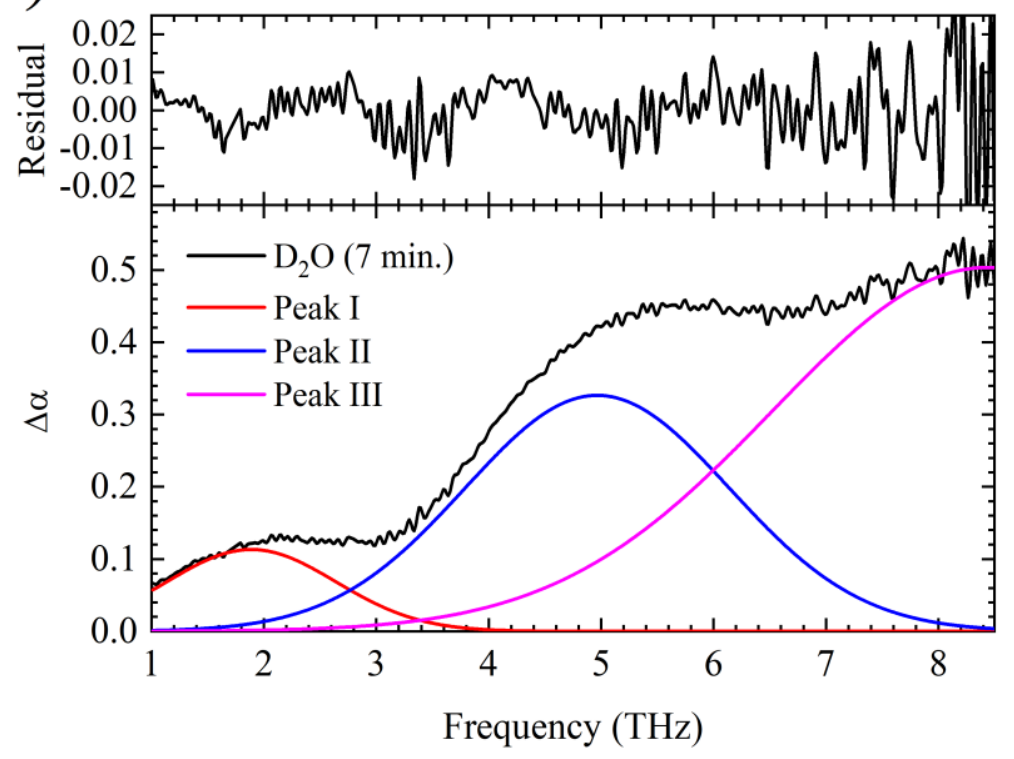

Figure 5 Black lines show (a) $\Delta \alpha$ of $\mathrm{H}_{2} \mathrm{O}$ observed at 10 minutes and (b) $\Delta \alpha$ of $\mathrm{D}_{2} \mathrm{O}$ observed at 7 minutes, respectively. Each spectrum is fitted by three Gaussian functions as Peak I, II and III.

The spectra shown in the upper panel show residual of fitting functions. 


\begin{tabular}{lcccccc}
\hline & \multicolumn{3}{c}{$\mathrm{H}_{2} \mathrm{O}$} & $\mathrm{D}_{2} \mathrm{O}$ \\
\hline & Peak I & Peak II & Peak III & Peak I & Peak II & Peak III \\
\hline Frequency (THz) & $1.81(1)$ & $5.02(7)$ & $7.78(8)$ & $1.89(1)$ & $4.97(4)$ & $8.43(4)$ \\
\hline FWHM (THz) & $1.67(4)$ & $3.01(8)$ & $3.4(2)$ & $1.76(4)$ & $2.77(8)$ & $4.5(4)$ \\
\hline Height & $0.110(1)$ & $0.34(2)$ & $0.32(1)$ & $0.113(1)$ & $0.32(3)$ & $0.504(3)$ \\
\hline
\end{tabular}

Table I Frequency, FWHM and peak height of Peak I, II and III fitted by the Gaussian functions

in Figure 4. The numbers in the parenthesis shows the uncertainties in the last digit.

Computational Simulations. In order to gain atomic-level insight into the dynamics occurring at terahertz frequencies in the hydrated materials, ab initio molecular dynamics (AIMD) and solid-state density functional theory (DFT) simulations were performed with CP2k. For the AIMD simulations, an initial simulation box containing eight symmetry independent polymer chains, each of which contained four polymer subunits, for a total of 608 independent atoms per cell. The system was subsequently hydrated to a $10 \% \mathrm{w} / \mathrm{w}$ concentration by randomly introducing 20 water molecules based on a minimum van der Waals radius cutoff methodology. The resulting system was equilibrated within the isothermal-isobaric (NPT) ensemble for $50 \mathrm{ps}$, which resulted in a bulk structure that strayed from crystallinity, at which point the canonical ensemble (NVT) was used in order to generate an infrared spectrum. The presented IR spectra are a result of taking the Fourier transform of the dipole moment autocorrelation function. 
The spectra show a general agreement with the experimental data, although the simulated spectra have more resolvable features than the experimental spectra. This is likely caused by the limitations of the utilized structural model, which imposes translational symmetry in order to make use of periodic boundary conditions. Thus, while the structure within the simulation cell is disordered, technological limitations hinder a fully-disordered model from being generated, resulting in a pseudo-crystalline simulation. Nonetheless, the simulations provide valuable insight into the structure and dynamics of both the bound waters, the polymer backbone, and the complex water-polymer dynamics, all of which can be characterized with atomic-level precision.

Figure 6 presents the result of the vibrational simulations. Particularly, the hydrated nylon spectrum shows significantly more absorption compared to the anhydrous spectra in the $0-3$ $\mathrm{THz}$ and $5-9 \mathrm{THz}$ regions, which is also observed in the experimental data. Also shown in Figure 6 is the difference between the wet and dry nylon spectra, which results in a small peak between $1-3 \mathrm{THz}$ and a large peak between $6-8 \mathrm{THz}$, in accordance with the peaks I and III in $\Delta \alpha$, respectively.

In order to assign the spectra, the average positions of the atoms over the last $500 \mathrm{fs}$ of the NVT trajectory were used as a starting point for geometry optimization calculations using static-DFT simulations with Cp2k. Upon complete minimization (within the constraint of a fixed unit cell), frequency calculations were performed within the harmonic approximation. 
Analysis of the vibrational normal modes (animations available in the ESI) corresponding to the three peak regions described previously provides insight into the water dynamics occurring at these frequencies. In the low-frequency region (below $3 \mathrm{THz}$ ), the observed motions correspond to highly-collective vibration of the water molecules in concert with much of the polymer backbone. While the motion of the water molecules is similar in nature to the previously described intermolecular bending in liquid water, the interaction with the polymer backbone leads to a diverse set of modes due to the large number of varying interactions that the water molecules exhibit in this system, with most modes involving external motions (i.e. hindered translations and rotations). Interestingly, a large majority of the observed motions only involve the weakly-bonded water molecules, i.e. those that exhibit either no hydrogen bonding or only a single hydrogen bonding interaction, while those that that are more strongly bound do not participate strongly in the lowest-frequency dynamics.

At higher frequencies, specifically those between approximately $3-6 \mathrm{THz}$, the dynamics occurring in the region involve more local motions that are simultaneously coupled to external librations. For example, a number of modes represent hindered translations and rotations with a simultaneous intramolecular component, such as an $\mathrm{OH}$ bond stretch. While this region involves all of the various water molecules, a majority of the modes consist of motions from the more strongly-bound waters, i.e. those having multiple hydrogen bonding interactions with the polymer 
helices. As the frequency of the transitions increase, the modes become increasingly more localized. For example, a major feature observed in the hydrated spectrum around $5.9 \mathrm{THz}$ corresponds to an intermolecular water libration coupled with a simultaneous $\mathrm{O}-\mathrm{H}$ bond stretch, as well as concomitant motion of the polymer backbone. The highest frequency modes, those occurring above $6 \mathrm{THz}$, involve much more localized motions of the water molecules, and specifically those waters that are strongly interacting with the polymer backbone via hydrogen bonds and spatial proximity. For example, one of the significant features occurring near $8.5 \mathrm{THz}$ involves concerted $\mathrm{H}-\mathrm{O}-\mathrm{H}$ bending and $\mathrm{O}-\mathrm{H}$ stretching along the hydrogen bonding coordinate between the water molecules and the polymer backbone.

The results of the simulations highlight that the motions of the water molecules are generally comparable between bulk water and the hydrated nylon system, in both mode-type and transition frequency. However, the introduction of the polymer backbone results in a highly diverse set of intermolecular interactions, which in turn yields a very large number of IR-active vibrational modes whose motions increase in complexity and become significantly more collective as frequency decreases. 


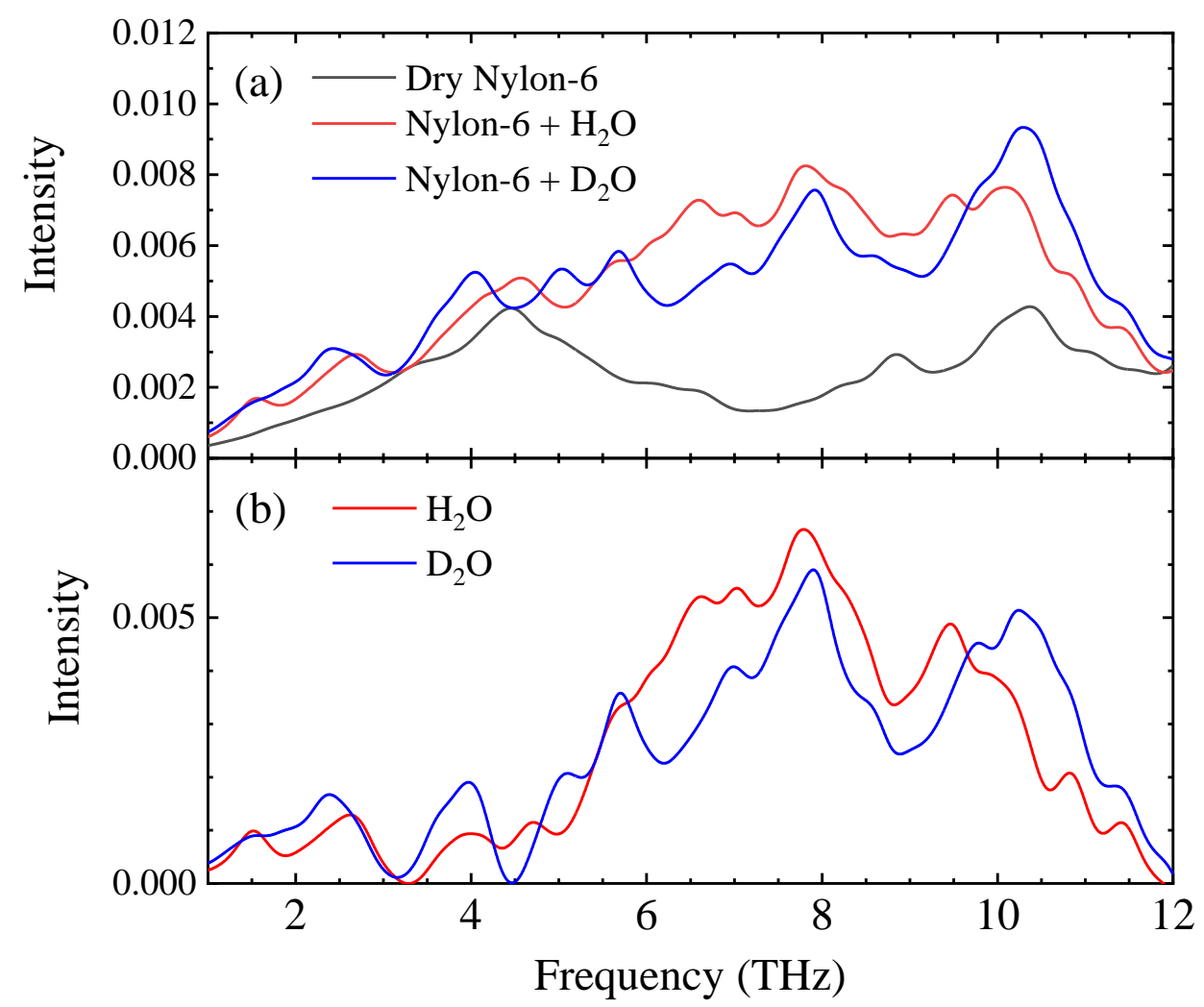

Figure 6 - (a) AIMD simulated THz spectra of nylon- 6 and those with bound water $\left(\mathrm{H}_{2} \mathrm{O}\right.$ and $\mathrm{D}_{2} \mathrm{O}$ ) (b) THz spectra of bound water obtained by the difference spectrum between hydrated and dehydrated nylon.

Intensity change with the water desorption. Figure 6 shows the intensity change of $\Delta \alpha$ of (a) $\mathrm{H}_{2} \mathrm{O}$ and (b) $\mathrm{D}_{2} \mathrm{O}$ during the desorption of water molecules. The intensities are taken at the center frequencies of the Peak I-III, respectively, and normalized with the values at the first data points. All the plots in the Fig. 7 shows similar decay with time as water content in the Fig. 2. The weight of water loses about $80 \%$ in 4 hours, and similarly, $\Delta \alpha$ 
decreases $65-80 \%$ in 4 hours. There is small difference of the decay rate between the Peak origins. The intensity of Peak I always decays faster than Peaks II and III. After 4 hours of desorption, the intensities differ by about $10 \%$. This result suggests that Peaks originates in different type of water molecules. This is in agreement with the ab initio simulations, which suggest Peak 1 has its origins in loosely bound water, while peaks 2 and 3 arise from more strongly bound molecules. 
(a)

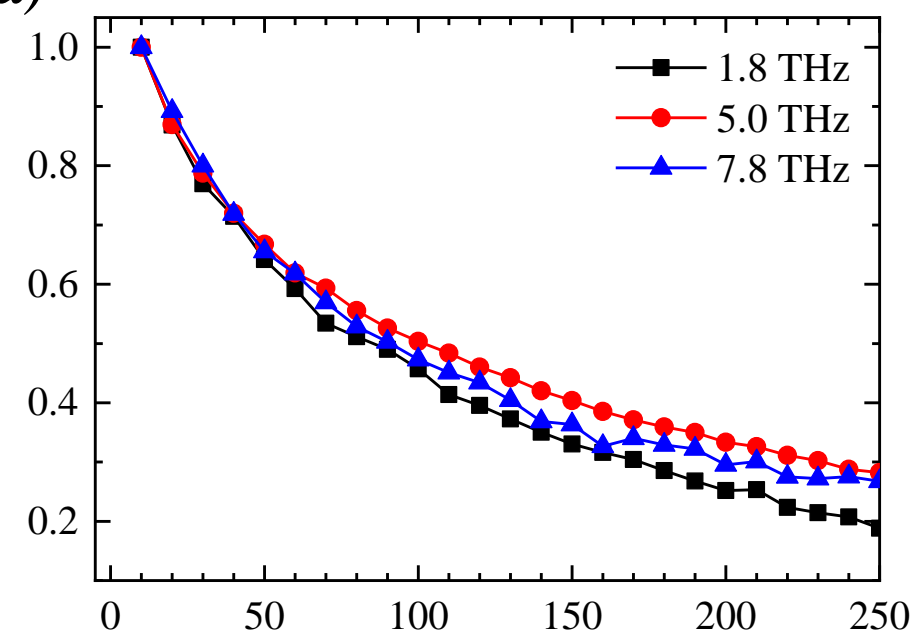

(b)

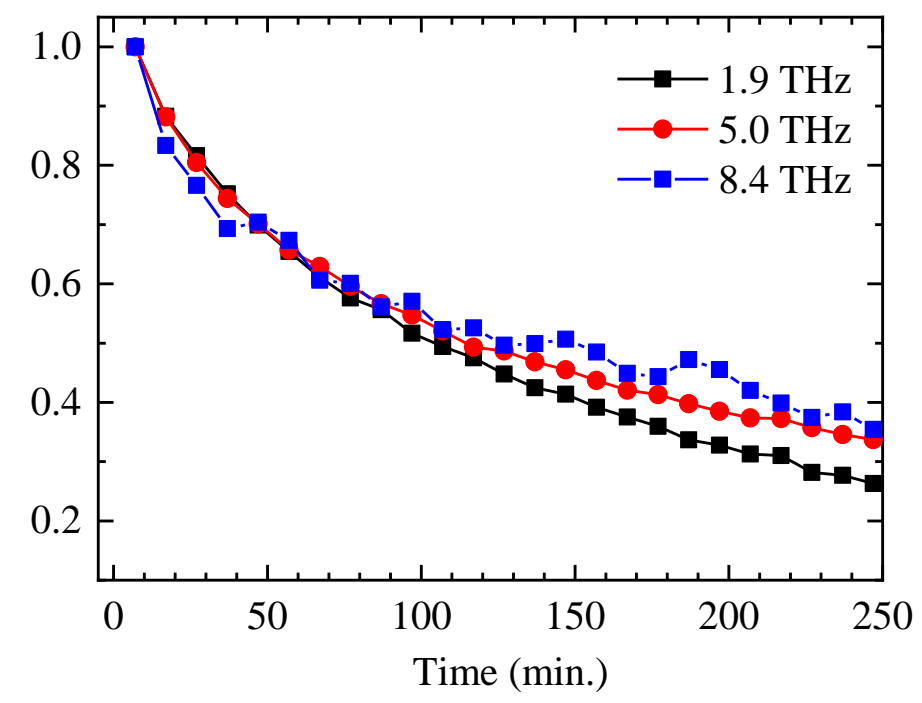

Figure 7 Intensity of the $\Delta \alpha$ of (a) $\mathrm{H}_{2} \mathrm{O}$ and (b) $\mathrm{D}_{2} \mathrm{O}$ during the desorption of water molecules. The intensities are taken at the center frequencies of the Peak I-III, respectively, and normalized with the values at the first data points.

\section{- CONCLUSION}

In conclusion, our results demonstrated the use of $\mathrm{THz}$ spectroscopy for the observation 
of water molecules adsorbed in the polymer films. The THz absorption spectra of nylon6 films drastically changes their intensity during the hydration and drying process. Also, the structural change from $\gamma$ to $\alpha$ crystals induced by the hydration was observed by the characteristic band of $\alpha$-nylon-6 at $6.5 \mathrm{THz}$. THz spectra of adsorbed water molecules in the nylon films were observed by the continuous measurement of $\alpha$-nylon during the dehydration. The differential spectra " $\Delta \alpha$ " clearly shows three absorption bands of water molecules named Peak I, II and III. By using both $\mathrm{H}_{2} \mathrm{O}$ and $\mathrm{D}_{2} \mathrm{O}$, the frequencies and changes upon dehydration were compared. Additioanlly, through the use of AIMD simulations, the spectral features could be assigned and interpreted with atomic-level precision. As a result, Peak II was assigned as the intermolecular stretching modes of water molecules. Peak I was the intermolecular bending vibrations strongly coupled with the vibration of the nylon chains. Peak III was the collective vibration of water molecules and polymer chains interacting with strong hydrogen bondings. Thus, detailed dynamics of the water molecules adsorbed in the nylon- 6 films are obtained by the $\mathrm{THz}$ spectroscopy and computational simulations. Since $\mathrm{THz}$ spectroscopy gives direct information of the intermolecular dynamics than other spectroscopic methods, it is expected to become a novel method for the polymer structural analysis. Furthermore, $\mathrm{THz}$ spectroscopy enables in situ measurement for large samples. Unlike DSC or NMR, non-invasive spectroscopic imaging is possible to detect the water distribution in polymer products. Therefore, 
we expect that $\mathrm{THz}$ spectroscopy may be used as a novel diagnosis tool for polymer industries in the near future.

\section{- Experimental Section}

Preparation of the nylon-6 films. Nylon-6 pellets (Sigma Aldrich) were melted on a hot plate at $250^{\circ} \mathrm{C}$ and pressed with an aluminum block for a few minutes. The obtained films were then cooled down to the room temperature. After the samples were vacuum dried at $40{ }^{\circ} \mathrm{C}$ for 1 week, THz spectra of dried samples were measured for references. Next, the sample films are stored in the humid environments at room temperature controlled by water and saturated solutions of inorganic salts for 2 weeks.

Measurement of THz spectra. $\mathrm{THz}$ absorption spectra were measured by a FT-FIR spectrometer (JASCO: FARIS). The film samples are placed on a sample holder which is tilted against THz beam path. The tilt angle is adjusted as Brewster's angle to minimize the multireflection inside the sample, which causes fringe in the absorption spectrum. Detail of the Brewster's angle sample holder is written in elsewhere. ${ }^{38} \mathrm{THz}$ absorption spectra were measured for 1-9 THz with a high-pressure mercury lamp as the light source, a wire-grid beam splitter, and a superconducting bolometer (QMC: QNbB/PTC). Each spectrum was measured with spectral resolution of $0.06 \mathrm{THz}$, accumulated for 360 scans. 
ab initio simulations. The freely available CP2k software package was used for all simulations, which made use of three-dimensional periodic boundary conditions. ${ }^{53,54}$ The PerdewBurke-Ernzerhof (PBE) density functional ${ }^{55}$ coupled with the dispersion correction of Grimme (Grimme-D ${ }^{56,57}$ ) and all atoms were represented using the double-zeta DZVP basis set. ${ }^{58}$ AIMD simulations were performed within the isobaric-isothermal (NPT) and canonical ensemble (NVT), with the temperature maintained at $300 \mathrm{~K}$ using a Nose-Hoover chain thermostat. ${ }^{59-61}$ The initial structure was based off the previously published experimental X-ray diffraction structure, ${ }^{62}$ with hydrogen positions added where appropriate. The NPT simulations were performed using a timestep of $1 \mathrm{fs}$, and were equilibrated for 20 ps prior to performing the NVT simulations. The NVT simulations were performed using a timestep of $0.5 \mathrm{fs}$, and were equilibrated for 5 ps prior to performing the production run for $30 \mathrm{ps}$ ( $35 \mathrm{ps}$ total). The molecular dipole moments were determined by calculating localized Wannier functions every $2.5 \mathrm{ps}$, and the resulting IR spectra for the simulation determined by taking the Fourier transform of the dipole moment autocorrelation function using the Travis postprocessing software, as previously described. ${ }^{63-65}$ The final structure (averaged from the last $500 \mathrm{fs}$ of the AIMD simulations) was used to initialize geometry optimizations, which were performed with a fixed unit cell. Static frequencies were determined within the harmonic approximation by numerical differentiation. ${ }^{66,67}$ 
- ASSOCIATED CONTENT

\section{Supporting Information}

The Supporting Information is available free of charge on the ACS Publications website at DOI: XXX

Representative animations of motions occurring in the three regions $(2.56 \mathrm{THz}$, $5.61 \mathrm{THz}$, and $7.94 \mathrm{THz})$.

\section{- AUTHOR INFORMATION}

\section{Corresponding Authors}

*hoshina@riken.jp

*Michael.Ruggiero@uvm.edu

\section{ORCID}

Hiromichi Hoshina: 0000-0002-4770-2005

Michael.Ruggiero: 0000-0003-1848-2565

Notes

The authors declare no competing financial interest.

\section{- ACKNOWLEDGMENTS}

MTR thanks the University of Vermont for its continued support. 
- REFERENCES

(1) Lee, Y.-S. Principle of Terahertz Science and Technology; Springer: New York, 2009. https://doi.org/10.1007/978-0-387-09540-0.

(2) Zhu, Z.; Mühlbacher, D.; Morana, M.; Koppe, M.; Scharber, M. C.; Waller, D.;

Dennler, G.; Brabec, C. J.; Petrova-Koch, V.; Hezel, R.; et al. Terahertz

Spectroscopy and Imaging; Peiponen, K.-E., Zeitler, A., Kuwata-Gonokami, M.,

Eds.; Springer Series in Optical Sciences; Springer Berlin Heidelberg: Berlin,

Heidelberg, Heidelberg, 2013; Vol. 171. https://doi.org/10.1007/978-3-642-

29564-5.

(3) Zhang, F.; Wang, H. W.; Tominaga, K.; Hayashi, M. Mixing of Intermolecular and Intramolecular Vibrations in Optical Phonon Modes: Terahertz Spectroscopy and Solid-State Density Functional Theory. Wiley Interdiscip. Rev. Comput. Mol. Sci. 2016, 6 (4), 386-409. https://doi.org/10.1002/wcms.1256.

(4) Zhang, F.; Wang, H. W.; Tominaga, K.; Hayashi, M. Characteristics of LowFrequency Molecular Phonon Modes Studied by THz Spectroscopy and SolidState Ab Initio Theory: Polymorphs I and III of Diflunisal. J. Phys. Chem. B 2016, 120 (8), 1698-1710. https://doi.org/10.1021/acs.jpcb.5b08798.

(5) Hurley, W. J.; Kuntz Jr., I. D.; Leroi, G. E. Far-Infrared Studies of Hydrogen 
Bonding. J. Am. Chem. Soc. 1966, 88 (14), 3199-3202.

(6) Nielsen, O. F. Low-Frequency Vibrational Spectra of Some Hydrogen-Bonded Amides. J. Mol. Struct. 1991, 242, 273.

(7) El Khoury, Y.; Hellwig, P. Far Infrared Spectroscopy of Hydrogen Bonding Collective Motions in Complex Molecular Systems. Chem. Commun. 2017, 53, 8389-8399. https://doi.org/10.1039/C7CC03496B.

(8) Walther, M.; Fischer, B. M.; Jepsen, P. U. Noncovalent Intermolecular Forces in Polycrystalline and Amorphous Saccharides in the Far Infrared. Chem. Phys. 2003, 288 (2-3), 261-268. https://doi.org/10.1016/S0301-0104(03)00031-4.

(9) Bershtein, V. A.; Ryzhov, V. A. Relationship between Molecular Characteristics of Polymers and Parameters of Far-Infrared Spectra. J. Macromol. Sci. Part B Phys. 1984, 23 (2), 271-287. https://doi.org/10.1080/00222348408219460.

(10) Bershtein, V.; Ryzhov, V. Far Infrared Spectroscopy of Polymers. Polym. Anal. Charact. 1994, 114, 43-121.

(11) Wietzke, S.; Jansen, C.; Jung, T.; Reuter, M.; Baudrit, B.; Bastian, M.; Chatterjee, S.; Koch, M. Terahertz Time-Domain Spectroscopy as a Tool to Monitor the Glass Transition in Polymers. Opt. Express 2009, 17 (21), 1900619014. 
(12) Wietzke, S.; Jansen, C.; Reuter, M.; Jung, T.; Kraft, D.; Chatterjee, S.; Fischer, B. M.; Koch, M. Terahertz Spectroscopy on Polymers: A Review of Morphological Studies. J. Mol. Struct. 2011, 1006 (1-3), 41-51. https://doi.org/10.1016/j.molstruc.2011.07.036.

(13) Wietzke, S.; Jansen, C.; Reuter, M.; Jung, T.; Hehl, J.; Kraft, D.; Chatterjee, S.; Greiner, A.; Koch, M. Thermomorphological Study of the Terahertz Lattice Modes in Polyvinylidene Fluoride and High-Density Polyethylene. Appl. Phys. Lett. 2010, 97 (2), 022901. https://doi.org/10.1063/1.3462312.

(14) Itoh, K.; Shimanouchi, T. Vibrational Frequencies and Modes of Alpha-Helix. Biopolymers 1970, 9 (4), 383-399. https://doi.org/10.1002/bip.1970.360090402.

(15) Ruggiero, M. T.; Sibik, J.; Orlando, R.; Zeitler, J. A.; Korter, T. M. Measuring the Elasticity of Poly- 1 -Proline Helices with Terahertz Spectroscopy. Angew. Chemie - Int. Ed. 2016, 55 (24), 6877-6881. https://doi.org/10.1002/anie.201602268.

(16) Hoshina, H.; Morisawa, Y.; Sato, H.; Minamide, H.; Noda, I.; Ozaki, Y.; Otani, C. Polarization and Temperature Dependent Spectra of Poly(3Hydroxyalkanoate)s Measured at Terahertz Frequencies. Phys. Chem. Chem. Phys. 2011, 13 (20), 9173-9179. https://doi.org/10.1039/c0cp02435j. 
(17) Hoshina, H.; Ishii, S.; Yamamoto, S.; Morisawa, Y.; Sato, H.; Uchiyama, T.;

Ozaki, Y.; Otani, C. Terahertz Spectroscopy in Polymer Research: Assignment of Intermolecular Vibrational Modes and Structural Characterization of Poly(3Hydroxybutyrate). IEEE Trans. Terahertz Sci. Technol. 2013, 3 (3), 248-258. https://doi.org/10.1109/TTHZ.2013.2253154.

(18) Yamamoto, S.; Morisawa, Y.; Sato, H.; Hoshina, H.; Ozaki, Y. Quantum Mechanical Interpretation of Intermolecular Vibrational Modes of Crystalline Poly-(R)-3-Hydroxybutyrate Observed in Low-Frequency Raman and Terahertz Spectra. J. Phys. Chem. B 2013, 117 (7), 2180-2187. https://doi.org/10.1021/jp309704k.

(19) Funaki, C.; Yamamoto, S.; Hoshina, H.; Ozaki, Y.; Sato, H. Three Different Kinds of Weak $\mathrm{C}-\mathrm{H} \cdots \mathrm{O}=\mathrm{C}$ Inter- and Intramolecular Interactions in $\operatorname{Poly}(\varepsilon-$ Caprolactone) Studied by Using Terahertz Spectroscopy, Infrared Spectroscopy and Quantum Chemical Calculations. Polym. (United Kingdom) 2018, 137, 245254. https://doi.org/10.1016/j.polymer.2018.01.025.

(20) Yamamoto, S.; Miyada, M.; Sato, H.; Hoshina, H.; Ozaki, Y. Low-Frequency Vibrational Modes of Poly(Glycolic Acid) and Thermal Expansion of Crystal Lattice Assigned On the Basis of DFT-Spectral Simulation Aided with a 
Fragment Method. J. Phys. Chem. B 2017, 121 (5), 1128-1138.

https://doi.org/10.1021/acs.jpcb.6b11304.

(21) Suzuki, H.; Ishii, S.; Sato, H.; Yamamoto, S.; Morisawa, Y.; Ozaki, Y.;

Uchiyama, T.; Otani, C.; Hoshina, H. Brill Transition of Nylon-6 Characterized by Low-Frequency Vibration through Terahertz Absorption Spectroscopy. Chem. Phys. Lett. 2013, 575, 36-39. https://doi.org/10.1016/j.cplett.2013.05.002.

(22) Suzuki, H.; Ishii, S.; Otani, C.; Hoshina, H. Low-Frequency Vibrations of Polyamide- 6 as a Function of Temperature and Thermal History Investigated by Terahertz Absorption Spectroscopy. Eur. Polym. J. 2015, 67, 284-291. https://doi.org/10.1016/j.eurpolymj.2015.04.009.

(23) Schweicher, G.; D’Avino, G.; Ruggiero, M. T.; Harkin, D. J.; Broch, K.; Venkateshvaran, D.; Liu, G.; Richard, A.; Ruzié, C.; Armstrong, J.; et al. Chasing the "Killer" Phonon Mode for the Rational Design of Low-Disorder, HighMobility Molecular Semiconductors. Adv. Mater. 2019, 1902407, 1902407. https://doi.org/10.1002/adma.201902407.

(24) Ruggiero, M. T.; Zhang, W.; Bond, A. D.; Mittleman, D. M.; Zeitler, J. A. Uncovering the Connection between Low-Frequency Dynamics and Phase Transformation Phenomena in Molecular Solids. Phys. Rev. Lett. 2018, 120 (19), 
196002. https://doi.org/10.1103/PhysRevLett.120.196002.

(25) Sibik, J.; Löbmann, K.; Rades, T.; Zeitler, J. A. Predicting Crystallization of Amorphous Drugs with Terahertz Spectroscopy. Mol. Pharm. 2015, 12 (8), 3062-3068. https://doi.org/10.1021/acs.molpharmaceut.5b00330.

(26) Hoshina, H.; Ishii, S.; Morisawa, Y.; Sato, H.; Noda, I.; Ozaki, Y.; Otani, C. Isothermal Crystallization of Poly(3-Hydroxybutylate) Studied by Terahertz Two-Dimensional Correlation Spectroscopy. Appl. Phys. Lett. 2012, 100, 011907. https://doi.org/10.1109/irmmw-THz.2011.6104771.

(27) Funaki, C.; Toyouchi, T.; Hoshina, H.; Ozaki, Y.; Sato, H. Terahertz Imaging of the Distribution of Crystallinity and Crystalline Orientation in a Poly( $\varepsilon$ Caprolactone) Film. Appl. Spectrosc. 2017, 71 (7), 1537-1542. https://doi.org/10.1177/0003702816684838.

(28) Zelsmann, H. R.; Condensie, M.; Moticulatre, S. Temperature Dependence of the Optical Constants for Liquid H2O and D2O in the Far IR Region. J. Mol. Struct. 1995, 350 (2), 95-114. https://doi.org/http://dx.doi.org/10.1016/00222860(94)08471-S.

(29) Nagai, M.; Yada, H.; Arikawa, T.; Tanaka, K. Terahertz Time-Domain Attenuated Total Reflection Spectroscopy in Water and Biological Solution. Int. 
J. Infrared Millimeter Waves 2006, 27 (4), 505-515.

https://doi.org/10.1007/s10762-006-9098-3.

(30) Yada, H.; Nagai, M.; Tanaka, K. The Intermolecular Stretching Vibration Mode in Water Isotopes Investigated with Broadband Terahertz Time-Domain Spectroscopy. Chem. Phys. Lett. 2009, 473 (4-6), 279-283.

https://doi.org/10.1016/j.cplett.2009.03.075.

(31) Shiraga, K.; t, k; Arikawa, T.; Saito, S.; Ogawa, Y. Reconsideration of the Relaxational and Vibrational Line Shapes of Liquid Water Based on Ultrabroadband Dielectric Spectroscopy. Phys. Chem. Chem. Phys. 2018, 20, 26200-26209. https://doi.org/10.1039/C8CP04778B.

(32) Jördens, C.; Wietzke, S.; Scheller, M.; Koch, M. Investigation of the Water Absorption in Polyamide and Wood Plastic Composite by Terahertz TimeDomain Spectroscopy. Polym. Test. 2010, 29 (2), 209-215. https://doi.org/10.1016/j.polymertesting.2009.11.003.

(33) Balakrishnan, J.; Fischer, B. M.; Abbott, D. Sensing the Hygroscopicity of Polymer and Copolymer Materials Using Terahertz Time-Domain Spectroscopy. Appl. Opt. 2009, 48 (12), 2262-2266. https://doi.org/10.1364/AO.48.002262.

(34) Yamamoto, N.; Ohta, K.; Tamura, A.; Tominaga, K. Broadband Dielectric 
Spectroscopy on Lysozyme in the Sub-Gigahertz to Terahertz Frequency

Regions: Effects of Hydration and Thermal Excitation. J. Phys. Chem. B 2016, 120 (21), 4743-4755. https://doi.org/10.1021/acs.jpcb.6b01491.

(35) Aoki, K.; Shiraki, K.; Hattori, T. Observation of Salt Effects on Hydration Water of Lysozyme in Aqueous Solution Using Terahertz Time-Domain Spectroscopy. Appl. Phys. Lett. 2013, 103 (17). https://doi.org/10.1063/1.4826699.

(36) Hishida, M.; Tanaka, K.; Yamamura, Y.; Saito, K. Cooperativity between Water and Lipids in Lamellar to Inverted-Hexagonal Phase Transition. J. Phys. Soc. Japan 2014, 83 (4), 1-8. https://doi.org/10.7566/JPSJ.83.044801.

(37) Shiraga, K.; Ogawa, Y.; Kondo, N. Hydrogen Bond Network of Water around Protein Investigated with Terahertz and Infrared Spectroscopy. Biophys. J. 2016, 111 (12), 2629-2641. https://doi.org/10.1016/j.bpj.2016.11.011.

(38) Hoshina, H.; Iwasaki, Y.; Katahira, E.; Okamoto, M.; Otani, C. Structure and Dynamics of Bound Water in Poly ( Ethylene-Vinylalcohol ) Copolymers Studied by Terahertz Spectroscopy. Polymer (Guildf). 2018, 148, 49-60. https://doi.org/10.1016/j.polymer.2018.06.020.

(39) Puffr, R.; Sebenda, J. On the Structure and Properties of Polyamides. XXVII. The Mechanism of Water Sorption in Polyamides. J. Polym. Sci. Part C 1967, 
$16,79-93$.

(40) Murthy, N. S.; Stamm, M.; Sibilia, J. P.; Krimm, S. Structural Changes

Accompanying Hydration in Nylon 6. Macromolecules 1989, 22 (3), 1261-1267.

https://doi.org/10.1021/ma00193a043.

(41) Murthy, N. S.; Akkapeddi, M. K.; Orts, W. J. Analysis of Lamellar Structure in

Semicrystalline Polymers by Studying the Absorption of Water and Ethylene

Glycol in Nylons Using Small-Angle Neutron Scattering. Macromolecules 1998,

31 (1), 142-152. https://doi.org/10.1021/ma9707603.

(42) Grubb, D. T.; Murthy, N. S. Real-Time X-Ray Study of Nylon-6 Fibers during

Dehydration: Equatorial Small-Angle Scattering Is Due to Surface Refraction.

Macromolecules 2010, 43 (2), 1016-1027. https://doi.org/10.1021/ma902083z.

(43) Laurati, M.; Sotta, P.; Long, D. R.; Fillot, L. A.; Arbe, A.; Alegrìa, A.; Embs, J.

P.; Unruh, T.; Schneider, G. J.; Colmenero, J.; et al. Dynamics of Water

Absorbed in Polyamides. Macromolecules 2012, 45 (3), 1676-1687.

https://doi.org/10.1021/ma202368x.

(44) Laurati, M.; Arbe, A.; Rios De Anda, A.; Fillot, L. A.; Sotta, P. Effect of Polar Solvents on the Crystalline Phase of Polyamides. Polymer (Guildf). 2014, 55 (12), 2867-2881. https://doi.org/10.1016/j.polymer.2014.04.031. 
(45) Reuvers, N. J. W.; Huinink, H. P.; Fischer, H. R.; Adan, O. C. G. Quantitative Water Uptake Study in Thin Nylon-6 Films with NMR Imaging. Macromolecules 2012, 45 (4), 1937-1945. https://doi.org/10.1021/ma202719x.

(46) Litvinov, V. M. Diffusivity of Water Molecules in Amorphous Phase of Nylon-6 Fibers. Macromolecules 2015, 48 (13), 4748-4753. https://doi.org/10.1021/acs.macromol.5b00570.

(47) Iwamoto, R.; Murase, H. Infrared Spectroscopic Study of the Interactions of Nylon-6 with Water. J. Polym. Sci. Part B Polym. Phys. 2003, 41 (14), 17221729. https://doi.org/10.1002/polb.10526.

(48) Fukuda, M.; Miyagawa, M.; Kawai, H.; Yagi, N.; Kimura, O.; Ohta, T. Fundamental Studies on the Interactions between Moisture and Textiles V. FT-IR Study on the Moisture Sorption Isotherm of Nylon 6. Polym. J. 2005, 19 (7), 785-804. https://doi.org/10.1295/polymj.19.785.

(49) Thomsen, B.; Kawakami, T.; Shigemoto, I.; Sugita, Y.; Yagi, K. WeightAveraged Anharmonic Vibrational Analysis of Hydration Structures of Polyamide 6. J. Phys. Chem. B 2017, 121 (24), 6050-6063. https://doi.org/10.1021/acs.jpcb.7b00372.

(50) Fukasawa, T.; Sato, T.; Watanabe, J.; Hama, Y.; Kunz, W.; Buchner, R. Relation 
between Dielectric and Low-Frequency Raman Spectra of Hydrogen-Bond Liquids. Phys. Rev. Lett. 2005, 95 (19), 197802.

https://doi.org/10.1103/PhysRevLett.95.197802.

(51) Korepanov, V.; Yu, C. C.; Hamaguchi, H. o. Hyper-Raman Investigation of Intermolecular Vibrations of Water and Ice. J. Raman Spectrosc. 2018, 49 (11), 1742-1746. https://doi.org/10.1002/jrs.5459.

(52) Okajima, H.; Ando, M.; Hamaguchi, H. O. Formation of "Nano-Ice" and Density Maximum Anomaly of Water. Bull. Chem. Soc. Jpn. 2018, 91 (6), 991-997. https://doi.org/10.1246/bcsj.20180052.

(53) Hutter, J.; Iannuzzi, M.; Schiffmann, F.; VandeVondele, J. Cp2k: Atomistic Simulations of Condensed Matter Systems. Wiley Interdiscip. Rev. Comput. Mol. Sci. 2014, 4 (1), 15-25. https://doi.org/10.1002/wcms.1159.

(54) VandeVondele, J.; Krack, M.; Mohamed, F.; Parrinello, M.; Chassaing, T.; Hutter, J. Quickstep: Fast and Accurate Density Functional Calculations Using a Mixed Gaussian and Plane Waves Approach. Comput. Phys. Commun. 2005, 167 (2), 103-128. https://doi.org/10.1016/j.cpc.2004.12.014.

(55) Perdew, J. P.; Burke, K.; Ernzerhof, M. Generalized Gradient Approximation Made Simple. Phys. Rev. Lett. 1996, 77 (18), 3865-3868. 
https://doi.org/10.1103/PhysRevLett.77.3865.

(56) Grimme, S.; Antony, J.; Ehrlich, S.; Krieg, H. A Consistent and Accurate Ab Initio Parametrization of Density Functional Dispersion Correction (DFT-D) for the 94 Elements H-Pu. J. Chem. Phys. 2010, 132 (15), 154104. https://doi.org/10.1063/1.3382344.

(57) Grimme, S.; Ehrlich, S.; Goerigk, L. Effect of the Damping Function in Dispersion Corrected Density Functional Theory. J. Comput. Chem. 2011, 32 (7), 1456-1465. https://doi.org/10.1002/jcc.21759.

(58) VandeVondele, J.; Hutter, J. Gaussian Basis Sets for Accurate Calculations on Molecular Systems in Gas and Condensed Phases. J. Chem. Phys. 2007, 127 (11), 114105. https://doi.org/10.1063/1.2770708.

(59) Nosé, S. A Unified Formulation of the Constant Temperature Molecular Dynamics Methods. J. Chem. Phys. 1984, 81 (1), 511-519. https://doi.org/10.1063/1.447334.

(60) NOSÉ, S. A Molecular Dynamics Method for Simulations in the Canonical Ensemble. Mol. Phys. 2002, 100 (1), 191-198. https://doi.org/10.1080/00268970110089108.

(61) Martyna, G. J.; Klein, M. L.; Tuckerman, M. Nosé-Hoover Chains: The 
Canonical Ensemble via Continuous Dynamics. J. Chem. Phys. 1992, 97 (4), 2635-2643. https://doi.org/10.1063/1.463940.

(62) Holmes, D. R.; Bunn, C. W.; Smith, D. J.; Chemical, I. The Crystal Structure of Polycaproamide : Nylon 6. J. Polym. Sci. 1955, 17 (84), 159-177. https://doi.org/10.1002/pol.1955.120178401.

(63) Brehm, M.; Kirchner, B. TRAVIS - A Free Analyzer and Visualizer for Monte Carlo and Molecular Dynamics Trajectories. J. Chem. Inf. Model. 2011, 51 (8), 2007-2023. https://doi.org/10.1021/ci200217w.

(64) Thomas, M.; Brehm, M.; Fligg, R.; Vöhringer, P.; Kirchner, B. Computing Vibrational Spectra from Ab Initio Molecular Dynamics. Phys. Chem. Chem. Phys. 2013, 15 (18), 6608. https://doi.org/10.1039/c3cp44302g.

(65) Ruggiero, M. T.; Zeitler, J. A. Resolving the Origins of Crystalline Anharmonicity Using Terahertz Time-Domain Spectroscopy and Ab Initio Simulations. J. Phys. Chem. B 2016, 120 (45), 11733-11739. https://doi.org/10.1021/acs.jpcb.6b10248.

(66) Zicovich-Wilson, C. M.; Pascale, F.; Roetti, C.; Saunders, V. R.; Orlando, R.; Dovesi, R. Calculation of the Vibration Frequencies of ?-Quartz: The Effect of Hamiltonian and Basis Set. J. Comput. Chem. 2004, 25 (15), 1873-1881. 
https://doi.org/10.1002/jcc.20120.

(67) Pascale, F.; Zicovich-Wilson, C. M.; López Gejo, F.; Civalleri, B.; Orlando, R.;

Dovesi, R. The Calculation of the Vibrational Frequencies of Crystalline

Compounds and Its Implementation in the CRYSTAL Code. J. Comput. Chem.

2004, 25 (6), 888-897. https://doi.org/10.1002/jcc.20019. 